\title{
Malignant Pleural Mesothelioma in a Patient with Systemic Sclerosis: The First Report
}

\author{
Navneet Singh ${ }^{a} \quad K^{2}$ aran Madan ${ }^{a}$ Anish Bhattacharyab ${ }^{b}$ Raje Nijhawan ${ }^{c}$ \\ Departments of a Pulmonary Medicine, ${ }^{b}$ Nuclear Medicine and ${ }^{c}$ Cytology and Gynaecological Pathology, \\ Postgraduate Institute of Medical Education and Research, Chandigarh, India
}

\section{Established Facts}

- Systemic sclerosis, in particular the diffuse cutaneous form, is associated with the occurrence of malignancies.

- The majority of cases of malignant pleural mesothelioma are associated with asbestos exposure.

\section{Novel Insights}

- Malignant pleural mesothelioma can occur in systemic sclerosis and without any asbestos exposure.

- Cases suspected as malignant pleural mesothelioma on clinicoradiological grounds must be confirmed by cyto/histopathological examination complemented by immunohistochemistry.

\section{Key Words}

Systemic sclerosis • Mesothelioma • Wilms tumor-1 antigen • Epithelial membrane antigen - Pleural fluid cytology

\footnotetext{
Abstract

Malignant pleural mesothelioma (MPM) is a relatively rare but aggressive pleural tumor. Systemic sclerosis (SSc) is associated with the development of lung cancer and other malignancies. We describe a 58-year-old never-smoker female, previously diagnosed with limited cutaneous SSc, who presented with chest pain on the left side, dyspnea and circum-
}

ferential nodular left pleural thickening. Fluorodeoxyglucose positron-emission tomography-computed tomography showed intense uptake in the thickened left pleura, mediastinal lymph nodes and left femur. Pleural fluid cytological examination along with nuclear and membranous positivity for Wilms tumor-1 antigen and epithelial membrane antigen on immunocytochemistry confirmed the diagnosis of MPM. To the best of our knowledge, this is the first report of MPM in a patient with SSC.

Copyright $\odot 2012$ S. Karger AG, Basel

\section{KARGER}

Fax +4161306 1234

E-Mail karger@karger.ch

www.karger.com (c) 2012 S. Karger AG, Basel

$0025-7931 / 13 / 0851-0066 \$ 38.00 / 0$

Accessible online at:

www.karger.com/res
Asst. Prof. Dr. Navneet Singh, MD, DM

Department of Pulmonary Medicine

Postgraduate Institute of Medical Education and Research (PGIMER)

Sector 12, Chandigarh 160012 (India)

Tel. +91 1722756 826, E-Mail navneetchd@yahoo.com 


\section{Introduction}

Pulmonary involvement is common among patients with systemic sclerosis (SSc) and tends to be either in the form of interstitial lung disease or pulmonary vascular disease [1]. Patients with diffuse cutaneous SSc have also been observed to have a higher incidence of malignancies including lung cancer [2]. However, occurrence of malignant pleural mesothelioma (MPM) amongst patients with SSc has never been reported before. We describe a woman with limited cutaneous SSc (LC-SSc) who developed MPM.

\section{Case Report}

A 58-year-old woman, a never-smoker, presented with insidious-onset left-sided chest pain and dyspnea which she had been having for 6 and 3 months, respectively. The pain was persistent, nonpleuritic in character, would awaken the patient from sleep and was partially relieved with analgesics. The dyspnea had increased progressively over 1 month, and at the time of presentation the patient was breathless at rest. There was no history of hemoptysis, wheezing or constitutional symptoms. She was a housewife without any history of asbestos exposure, chest trauma or exposure to radiation. There was also no history of asbestos exposure or of malignancies among any of her family members.

Two years ago, LC-SSc had been diagnosed during medical evaluation for symptoms of Raynaud's phenomenon and skintightening that had been present for 8 and 2 years, respectively. Historically, skin involvement was limited to the fingers, hands and face. Systemic hypertension had been detected 15 years previously and was controlled with amlodipine. There was no other significant personal history.

On examination, thickening of the skin on the fingers of both hands (fig. 1c) was observed. Opening of the mouth was limited by facial skin involvement (fig. 1a, b). Pulse rate was 104/min, blood pressure $160 / 90 \mathrm{~mm} \mathrm{Hg}$ and respiratory rate $30 / \mathrm{min}$. Oxygen saturation while breathing room air was $92 \%$ by pulse oximetry. Upon examination of the respiratory system, the trachea was central and the movements of the left hemithorax were reduced. There was a stony dull percussion note and a marked reduction in breath sound intensity over the left infrascapular, axillary and mammary areas. On the right side, end-inspiratory 'velcro' crackles were audible in the basal areas. Apart from this, the general physical and systemic examination was unremarkable.

A posteroanterior chest radiograph demonstrated an opaque left hemithorax without mediastinal shift. A high-resolution computed tomography (HRCT) scan of the thorax showed parenchymal septal thickening in both lower lobes (fig. 2a). On the mediastinal windows of the contrast-enhanced CT scan (fig. 2b), circumferential nodular and mass-like thickening (measuring approx. 2.5 $\mathrm{cm}$ ) of the left parietal pleura was observed. Mediastinal pleural involvement and multiloculated pleural collection were also seen. No obvious lung mass or nodules or thrombi in the pulmonary vasculature were visualized. Fluorine-18 $\left(18^{\mathrm{F}}\right)$ fluorodeoxyglucose (FDG) positron-emission tomography-computed tomography (PET-CT) demonstrated intense uptake in the thickened nodular
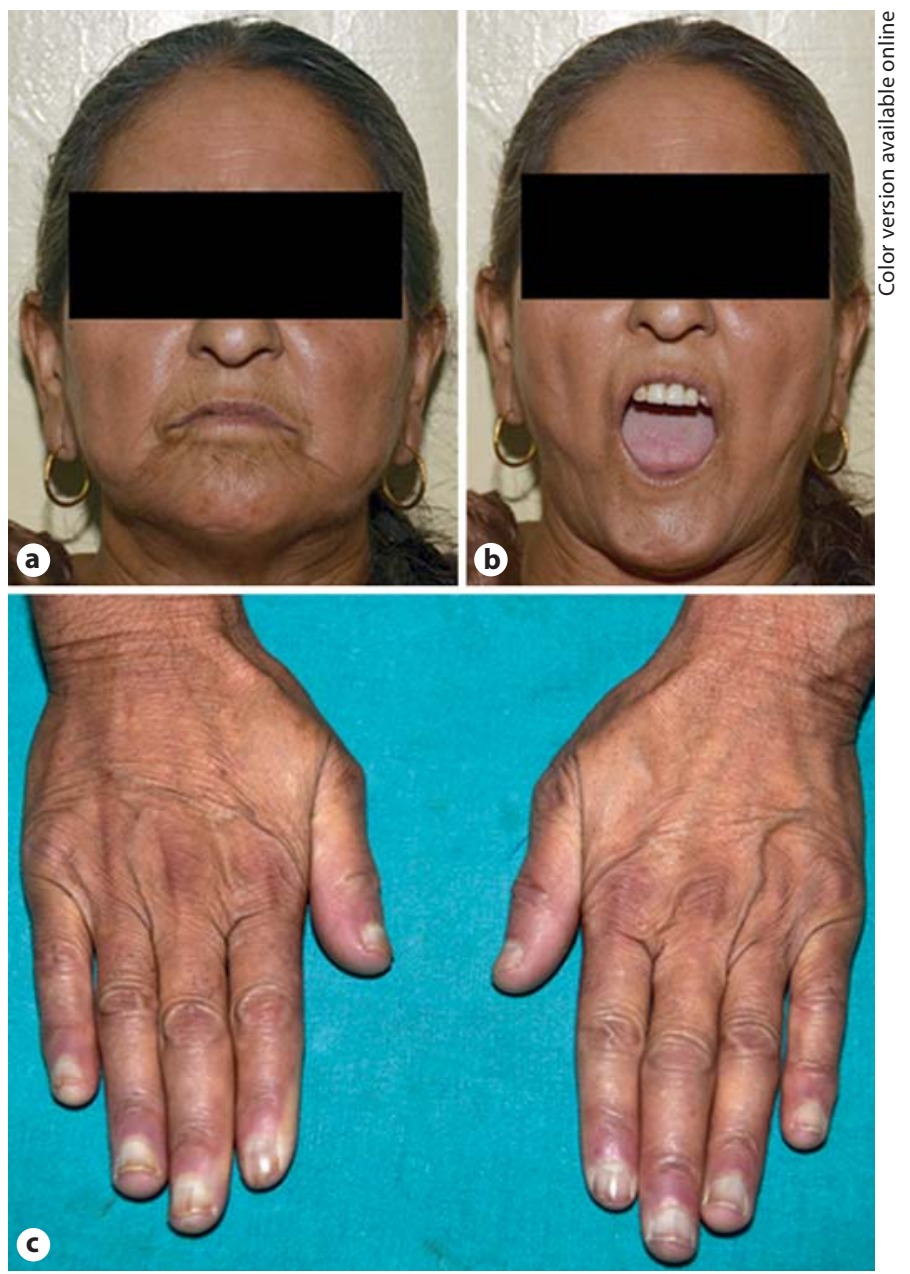

Fig. 1. a, b Clinical photograph depicting thickening of the skin on the face and restriction of the opening of the mouth. c Tightening of the skin and a shiny appearance of the fingers of both hands were observed up to the proximal interphalyngeal joints.

left pleura [maximum standardized uptake value $\left(\mathrm{SUV}_{\max }\right)$ 8.7] and mediastinal lymph nodes [highest mediastinal, right paratracheal, aortopulmonary window, prevascular $\left(\mathrm{SUV}_{\max } 5.2\right)$ and subcarinal] (fig. 2c). Moderate FDG uptake was also seen in multiple enlarged, bilateral supraclavicular and left-axillary lymph nodes. In addition, a focus of FDG uptake was seen in the intertrochanteric region of the left femur on the maximum-intensity projection image (fig. 2d). The clinicoradiological impression was that of MPM.

Ultrasound-guided thoracentesis drained straw-colored pleural fluid which analysis showed to be an exudate. Cytological examination of pleural fluid specimens showed malignant cells arranged in a glandular pattern with moderate pleomorphism, a high nuclear:cytoplasmic ratio, opened-up chromatin, conspicuous nucleoli and a moderate amount of vacuolated cytoplasm (fig. 3a). Immunocytochemistry showed nuclear and membranous positivity for Wilms tumor-1 antigen (WT-1) and epithelial membrane antigen (EMA), respectively (fig. $3 b, c)$. The patient 

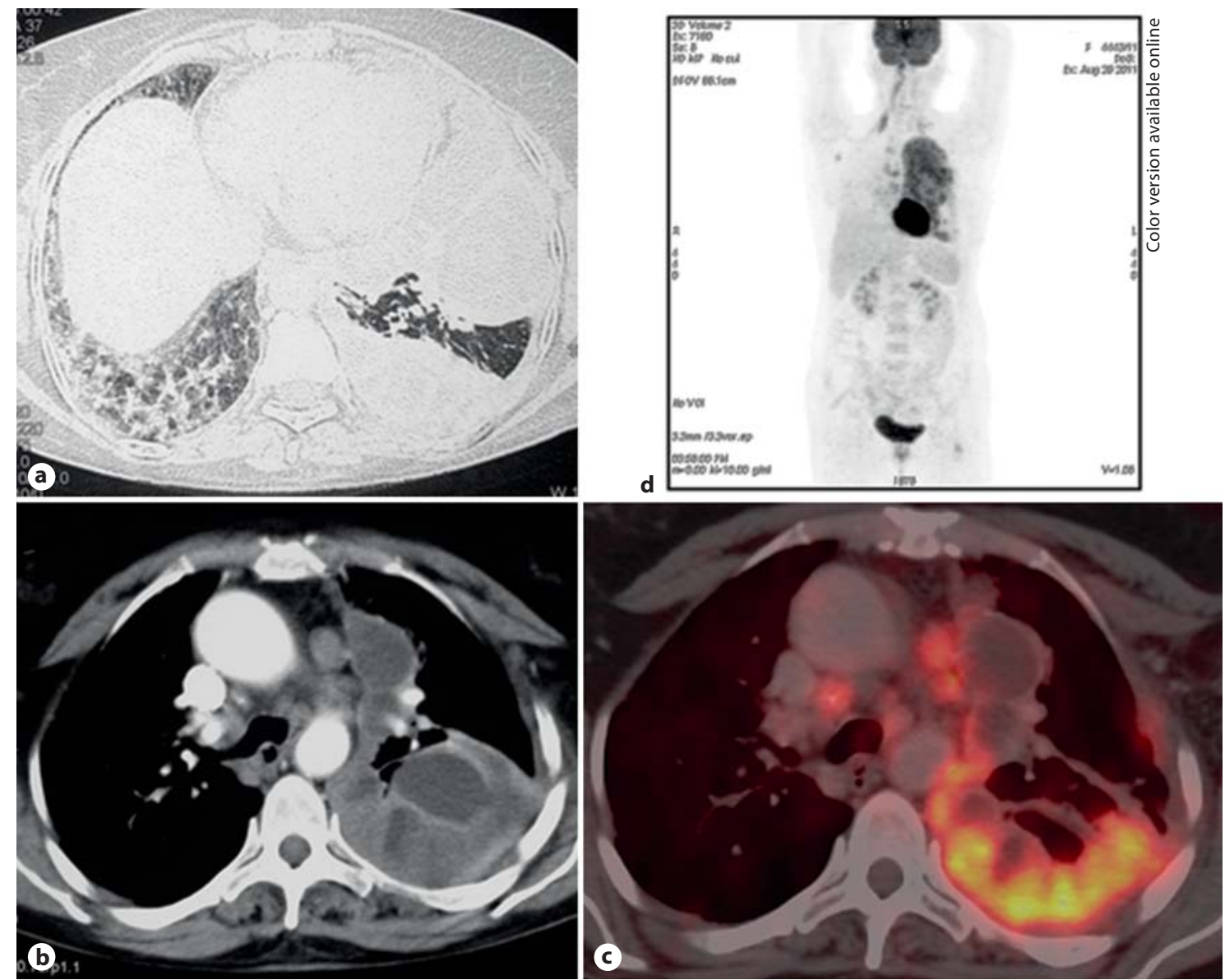

Fig. 2. a HRCT scan of the thorax showing parenchymal septal thickening in both lower lobes. b Contrast-enhanced CT examination of the thorax shows circumferential nodular mass-like thickening (measuring approx. $2.5 \mathrm{~cm}$ ) of the left parietal pleura. Mediastinal pleural involvement and multiloculated pleural collection are also seen. c $18^{\mathrm{F}}$-FDG PET-CT image showing intense uptake in the thickened nodular left pleura $\left(\mathrm{SUV}_{\max } 8.7\right)$ and me- diastinal lymph nodes [highest mediastinal, right paratracheal, aorto-pulmonary window, prevascular $\left(\mathrm{SUV}_{\max } 5.2\right)$ and subcarinal]. Moderate FDG uptake was also seen in multiple enlarged bilateral supraclavicular and left axillary lymph nodes. $\mathbf{d}$ In addition, a focus of FDG uptake was seen in the intertrochanteric region of the left femur on the maximum-intensity projection image. refused to give her consent for pleural biopsy. A final diagnosis was established of LC-SSc with stage IV (T2N3M1) MPM.

Drainage of the largest loculus was achieved using a pleural pigtail catheter while other loculi were small and multi-septated and thus not amenable for drainage. The catheter was removed after 5 days. The patient was subsequently started on chemotherapy [pemetrexed $\left(500 \mathrm{mg} / \mathrm{m}^{2}\right)$ and cisplatin $\left(65 \mathrm{mg} / \mathrm{m}^{2}\right)$ each on day 1 of a 3 -weekly cycle schedule] along with best supportive care.

\section{Discussion}

MPM is a relatively rare but aggressive tumor arising from the mesothelial lining of pleura. Malignant mesotheliomas can also arise from other serosal surfaces like the peritoneum, pericardium and tunica vaginalis [3]. The main carcinogenic agent implicated in the pathogenesis of MPM is exposure to asbestos. Increased exposure to asbestos during the last century has been implicated as the most important factor for its increasing incidence globally. Worldover, MPM has generated a lot of interest and concern since millions of people have been exposed to asbestos and a large number of cases of MPM are likely to be encountered globally in the years to come. The latent period between exposure to asbestos and the development of MPM is usually between 20 and 60 years. Infection with Simian virus 40 which is potentially oncogenic and acts by blockade of tumor suppressor genes is implicated as a cofactor in the pathogenesis of MPM. The 

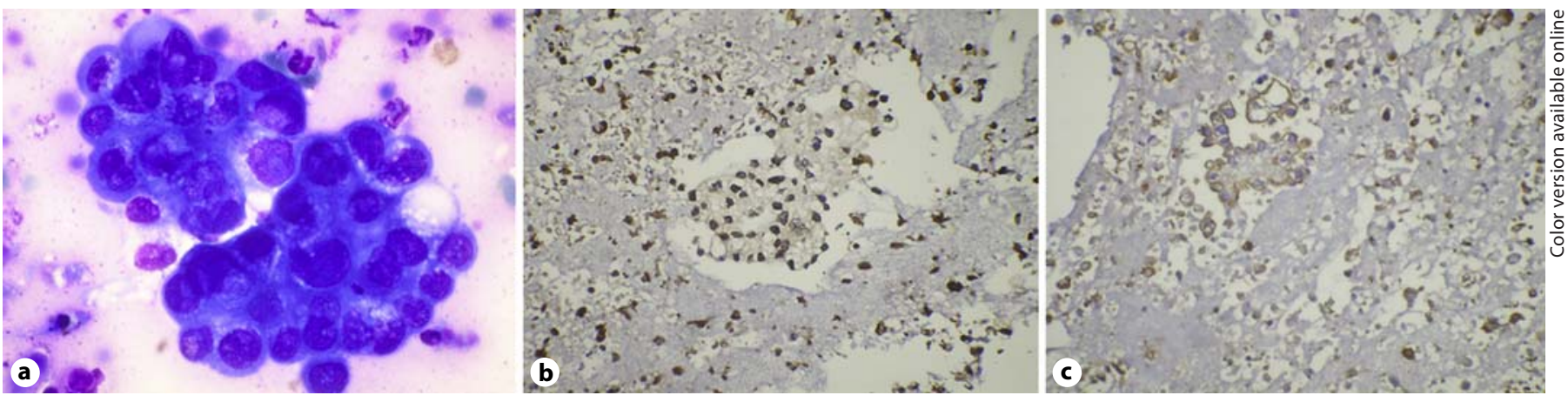

Fig. 3. a Cytological examination of the pleural fluid specimens showing malignant cells with moderate pleomorphism, a high nuclear:cytoplasmic ratio, opened-up chromatin, conspicuous nucleoli and a moderate amount of vacuolated cytoplasm. The cells were arranged in glandular pattern as well as lying singly. Immunocytochemistry of malignant cells shows nuclear positivity for WT-1 (b) and membranous positivity for EMA (c).

other recognized causes of mesothelioma include erionite exposure, ionizing radiation and chest injuries [4].

Most of the patients with MPM are male (80-85\%) and risk increases with age. It is important to mention that a history of occupational or paraoccupational exposure to asbestos may be lacking in up to $50 \%$ of female patients [5-6]. The usual presenting features include pleural effusion, chest pain and dyspnea, and are seen in around 60\% of patients [7]. A diagnosis of MPM should be suspected in any patient with pleural effusion and chest wall pain. Fewer than $30 \%$ of patients have constitutional symptoms at presentation, which do, however, eventually develop in most of the patients during the advanced stages of the disease [3]. Patients usually present when the disease has progressed somewhat, because extensive involvement of the pleural surfaces may occur without (significantly) compromising the patient physiologically and the average time interval between symptom onset and diagnosis is $2-3$ months [4]. Infrequently, the disease may be diagnosed incidentally on chest radiographs. Local invasion of contiguous structures is the usual cause for symptom progression and systemic metastases are unusual causes for death $[3,7]$. Uncommonly, the disease may involve the contralateral pleural cavity and peritoneum with miliary spread of the disease also being a reported manifestation [8].

Chest radiograph usually demonstrates pleural effusion or pleural-based masses. CT scans may demonstrate these encircling the underlying lung in a rind-like fashion. Chest wall invasion, though uncommon, is usually seen following a needle-intervention procedure and tumor seeding can occur through the tract. Magnetic reso- nance imaging can help in the assessment of invasion of the diaphragm, ribs or spinal canal [9]. PET-CT examination is a particularly useful imaging and staging modality, as it can show the extent of extra-thoracic disease [10]. High SUV correlates with a worse prognosis and PET-CT is the preferred imaging modality for response assessment following the administration of chemotherapy [3].

The most common and important issue in pathological diagnosis is the differentiation of mesothelioma from adenocarcinoma, particularly when pleural invasion has occurred in the latter. Cytopathological analysis of fluid obtained from pleural aspiration or fine-needle aspiration cytology samples is sufficient to establish a quick and accurate diagnosis, especially with the aid of immunocytochemistry. The first step is to establish the mesothelial origin of the tumor cells by using a marker such as WT-1. The second step is to establish that the cells are malignant, using a marker like EMA [3]. A confident diagnosis of MPM can be established in $80 \%$ of the cases upon analysis of the cytopathological samples alone [11]. Recently issued European guidelines for the management of MPM recommend performing histopathological examination of biopsied tissue for establishing the diagnosis of MPM. They also list the immunohistochemical markers that help in differentiating MPM from pulmonary adenocarcinoma and other malignancies [12]. In the index case, refusal by the patient to give consent precluded us from obtaining a pleural biopsy and performing immunohistochemistry with a wider panel of markers. However, at the same time, we would like to mention that as per the above mentioned guidelines, the presence of membranous positivity for EMA differentiates epithelioid MPM 
from adenocarcinoma where EMA positivity, if present, is cytoplasmic. Similarly, nuclear positivity for WT-1 is seen in MPM, while in adenocarcinoma WT-1 immunostaining is negative. In the index patient being reported by us, both membranous positivity for EMA as well as nuclear positivity for WT-1 were observed. We therefore feel that this characteristic immunocytochemical profile combined with the highly suggestive clinicoradiological picture helped us make a confident diagnosis of MPM in the absence of a pleural biopsy specimen.

A delay in diagnosis is usual in many cases, despite the ease by which cytopathological examinations of pleural fluid/tissue samples can enable a quick and accurate diagnosis. This scenario is more likely at centers where physicians and pathologists do not frequently come across this condition. The role of serum biomarkers has received attention in recent years. Measurement of serum mesothelin-related protein (SMRP), which is one of the beststudied biomarkers in MPM, has been proposed as a useful adjunct to pathological examination of samples in the diagnosis. In addition, serum SMRP-level measurement has been found to be useful in monitoring response to therapy $[3,13]$.

The likelihood of survival of patients with MPM beyond 1 year following diagnosis is 50\% and virtually all forms of treatment modalities are associated with poor eventual outcomes [4]. The tumor is resistant to conventional radiotherapy approach. Palliative chemotherapy is a feasible and beneficial option in patients with metastatic disease and good performance status. Chemotherapy with pemetrexed and cisplatin has been shown to be associated with improved median survival [14]. The role of aggressive surgery is controversial. Extra-pleural pneumonectomy is the most commonly performed surgical technique and should be a part of a multimodality ap- proach (in combination with radiotherapy, chemotherapy or other forms of treatment). A number of therapeutic approaches are being investigated including immunotherapy, gene therapy, tyrosine kinase inhibitors, VEGF inhibitors, photodynamic therapy, proteosome inhibitors and histone deacetylase inhibitors [3-4].

The association between SSc and malignancy is well recognized and the average risk for the development of cancer in SSc is 6.3\% (3.6-10.7\%). Risk factors include female sex, increasing age and diffuse cutaneous SSc. In various series, lung cancer (commonest) and breast cancer are the most frequently reported types of malignancy in patients with SSc, while other malignancies observed include gastrointestinal and urogenital cancers as well as lymphoid malignancies [2]. In a previous report, a patient with SSc who was suspected of having MPM, but in whom the diagnosis could not be established antemortem was ultimately shown to have pseudomesotheliomatous adenocarcinoma based upon the immunohistochemical profile on the autopsy specimen which included negative WT-1 immunostaining [15]. Thus, to the best of our knowledge, there is no previous report of a case of MPM in a patient with SSc. The average duration from the time of development of SSc to the development of lung cancer varies from 5 to 9 years while duration for other cancers is typically 2 years or less.

In summary, the index patient represents the first-ever reported case of MPM in a patient with SSc. Notable features include the fact that the patient was a female while MPM is commoner in males, and that the patient had LC-SSc whereas malignancy is more common with diffuse cutaneous SSc. Patients with SSc should be closely followed up and monitored for signs of the development of malignancy.

\section{References}

1 Woodhead F, Wells AU, Desai SR: Pulmonary complications of connective tissue diseases. Clin Chest Med 2008;29:149-164.

2 Wooten M: Systemic sclerosis and malignancy: A review of the literature. South Med J 2008;101:59-62.

3 Robinson BW, Lake RA: Advances in malignant mesothelioma. N Engl J Med 2005;353: 1591-1603.

-4 Robinson BW, Musk AW, Lake RA: Malignant mesothelioma. Lancet 2005;366:397408.
5 Magnani C, Terracini B, Ivaldi C, Botta M, Mancini A, Andrion A: Pleural malignant mesothelioma and non-occupational exposure to asbestos in Casale Monferrato, Italy. Occup Environ Med 1995;52:362-367.

6 Haber SE, Haber JM: Malignant mesothelioma: a clinical study of 238 cases. Ind Health 2011;49:166-172.

7 Lee YC, Light RW, Musk AW: Management of malignant pleural mesothelioma: a critical review. Curr Opin Pulm Med 2000;6: 267-274.
8 Purek L, Laroumagne S, Dutau H, Maldonado F, Astoul P: Miliary mesothelioma: a new clinical and radiological presentation in mesothelioma patients with prolonged survival after trimodality therapy. J Thorac Oncol 2011;6:1753-1756.

9 Wang ZJ, Reddy GP, Gotway MB, Higgins CB, Jablons DM, Ramaswamy M, Hawkins RA, Webb WR: Malignant pleural mesothelioma: evaluation with CT, MR imaging, and PET. Radiographics 2004;24:105-119. 
10 Flores RM, Akhurst T, Gonen M, Larson SM, Rusch VW: Positron emission tomography defines metastatic disease but not locoregional disease in patients with malignant pleural mesothelioma. J Thorac Cardiovasc Surg 2003;126:11-16.

11 Whitaker D, Henderson DW, Shilkin KB: The concept of mesothelioma in situ: implications for diagnosis and histogenesis. Semin Diagn Pathol 1992;9:151-161.
2 Scherpereel A, Astoul P, Baas P, Berghmans $\mathrm{T}$, Clayson $\mathrm{H}$, de Vuyst P, Dienemann $\mathrm{H}$, Galateau-Salle F, Hennequin C, Hillerdal G, Le Pechoux C, Mutti L, Pairon JC, Stahel R, van Houtte P, van Meerbeeck J, Waller D, Weder W: Guidelines of the European Respiratory Society and the European Society of Thoracic Surgeons for the management of malignant pleural mesothelioma. Eur Respir J 2010;35:479-495.

13 Robinson BW, Creaney J, Lake R, Nowak A, Musk AW, de Klerk N, Winzell P, Hellstrom KE, Hellstrom I: Mesothelin-family proteins and diagnosis of mesothelioma. Lancet 2003; 362:1612-1616.
14 Vogelzang NJ, Rusthoven JJ, Symanowski J, Denham C, Kaukel E, Ruffie P, Gatzemeier U, Boyer M, Emri S, Manegold C, Niyikiza C, Paoletti P: Phase III study of pemetrexed in combination with cisplatin versus cisplatin alone in patients with malignant pleural mesothelioma. J Clin Oncol 2003;21:26362644.

15 Yoshimi R, Takeno M, Yamanaka S, Shiina M, Kirino Y, Takeda Y, Sekiguchi A, Kobayashi $\mathrm{H}$, Ihata A, Motoji K, Ohno S, Ueda A, Soga T, Ishigatsubo Y: Systemic sclerosis and pseudomesotheliomatous adenocarcinoma of the lung. Mod Rheumatol 2006;16: 165-168. 